\title{
Opioid-Sparing Multimodal Analgesia Protocol for Lumpectomy Patients Results in Superior Postoperative Pain Control
}

\author{
Claudya Morin, MD ${ }^{1}$, Yamini Patel, MD ${ }^{1}$, Munazza Javid, MD ${ }^{1}$, Sarah E. Tevis, MD, FACS ${ }^{2}$, \\ Thais Fortes, MD $^{3}$, Peter Flom, MSc $^{4}$, Charusheela Andaz, MD, FACS ${ }^{1}$, Donna-Marie Manasseh, MD, FACS ${ }^{1}$, \\ Patrick Borgen, MD ${ }^{1}$, and Kristin E. Rojas, MD, FACOG, FACS ${ }^{5}$ \\ ${ }^{1}$ Department of Surgery, Maimonides Medical Center, Brooklyn, NY; ${ }^{2}$ Department of Surgery, University of Colorado \\ Anschutz Medical Campus, Aurora, CO; ${ }^{3}$ Sparrow Hospital, Lansing, MI; ${ }^{4}$ Peter Flom Statistical Consulting, New York \\ City, NY; ${ }^{5}$ Dewitt-Daughtry Department of Surgery, University of Miami Miller School of Medicine, Miami, FL
}

\begin{abstract}
Background. We sought to determine if lumpectomy patients who received perioperative opioid-sparing multimodal analgesia reported less pain when compared with those who received traditional opioid-based care.

Study Design. A prospective cohort of patients undergoing lumpectomy who received an opioid-sparing multimodal analgesia protocol [no opioids group (NOP)] was compared with a large cohort of patients who received traditional care [opioids group (OG)]. In-hospital and discharge opioids were compared using oral morphine equivalents (OMEs). Postoperative day one and week one pain scores were compared using the Kruskal-Wallis test. Results. Overall, 1153 patients underwent lumpectomy: 634 patients received the protocol (NOP), and 519 patients did not (OG). Median pain scores were significantly lower in the NOP cohort when compared with the OG cohort the day after surgery ( 2 vs. $0, p<0.001)$ and the week after surgery ( 1 vs. $0, p<0.001$ ). NOP patients were significantly less likely to report severe pain (7-10 on a 10-point scale) the day after surgery compared with OG patients $(15.7 \%$ vs. $6.9 \%, p=0.004)$. Patients in the NOP cohort were discharged with a median of zero OMEs (range $0-150$ ), while patients in the OG were discharged with a median of 90 OMEs (range $0-360 ; p<0.001$ ).
\end{abstract}

(C) Society of Surgical Oncology 2021

First Received: 29 January 2021

Accepted: 3 March 2021;

Published Online: 2 June 2021

P. Borgen, MD

e-mail: PBorgen@maimonidesmed.org
Conclusion. Implementation of an opioid-sparing multimodal analgesia protocol for lumpectomy patients resulted in superior pain control without a routine opioid prescription. Surgeons can improve their own patients' outcomes while addressing the larger societal issue of the opioid crisis by adopting similar protocols that decrease the quantity of opioids available for diversion.

The American Cancer Society reports that more than 100,000 breast-conserving surgeries are performed in the US each year. ${ }^{1}$ Historically, patients undergoing lumpectomy are discharged with an opioid prescription, but only $15-25 \%$ of the prescribed opioids are actually taken by the patient. ${ }^{2}$ This overprescription of opioids leads to a large quantity available for diversion, which has motivated surgical societies to reassess the necessity of postoperative opioids. $^{3}$

While opioids have historically been the gold standard in treating postoperative pain, they are associated with a risk of opioid use disorder and more recent data suggest that they can adversely impact patient care. Opioids lead to disrupted immune response and may contribute to poor oncologic outcome through immunosuppressive or inflammatory mechanisms. ${ }^{4,5}$ Incorporation of multimodal analgesia through breast surgery perioperative pain protocols leads to decreased opioid prescribing without an increased risk of complications. ${ }^{6}$ Previous work demonstrated that reflex opioid prescription after breast surgery could be eliminated without compromising postoperative pain. ${ }^{7}$ By combining a non-randomized pilot study with a large historical cohort, postoperative pain scores were compared to determine whether a multimodal analgesia 
protocol results in equivalent or improved pain control in patients undergoing one of the most common surgical procedures in the US. ${ }^{1}$

\section{METHODS}

A prospective pilot study of patients undergoing breast surgery at a single institution was designed and implemented after hospital Ethics Committee approval was obtained (IRB 2017-09-29). The elements of the opioidsparing protocol for patients undergoing lumpectomy and mastectomy without reconstruction have been previously described. ${ }^{7,8}$ The non-opioid (NOP) protocol utilizes multimodal analgesia, including gabapentin (GABA), acetaminophen (APAP), liposomal bupivacaine, and nonsteroidal anti-inflammatory drugs (NSAIDs). It can be simplified into three phases: preoperative education and medication, intraoperative non-opioid adjuncts including ketorolac $15 \mathrm{mg}$, and postoperative scheduled acetaminophen and ibuprofen (Fig. 1).

Preoperative education was enhanced with expanded counseling and written material that encouraged patients to purchase over-the-counter acetaminophen and ibuprofen prior to the day of surgery. Preoperative oral medications (acetaminophen $975 \mathrm{mg}$ and gabapentin $300 \mathrm{mg}$ ) were administered in the holding area with a sip of water. At least $20 \mathrm{cc}$ of long-acting local analgesia was applied through pre-incision infiltration of the skin, along with coverage of the lumpectomy cavity (including all five walls). Additional infiltration was performed pre-closure to the axillary subcutaneous tissue and the drain site.

Patients included in the prospective pilot study were compared with a large historical cohort of patients undergoing surgery approximately 2 years prior [opioid group (OG)]. Pilot study patients who did and did not receive the opioid-sparing protocol (September 2017-April 2019) were included along with the historical cohort (July 2015June 2016) that did not receive the protocol. Patients in the historical cohort and those receiving usual care (OG cohort) in the pilot study were routinely prescribed opioids at discharge according to provider preference. Those undergoing surgery between July 2016 and August 2017 were excluded since this represented a transitional period where different elements of the protocol were implemented inconsistently, such as the use of liposomal bupivacaine (Fig. 2). The present analysis included patients undergoing wire-localized lumpectomy, wireless localized lumpectomy, excisional biopsy, and re-excision with varied axillary management. Patients undergoing mastectomy or axillary surgery alone were excluded. Patients with selfreported opioid dependence or chronic opioid prescriptions noted in the patient medication list during the preoperative visit were also excluded from the present analysis.

Patient demographics, protocol adherence, and surgical outcomes were collected retrospectively and stored using the REDCap (Research Electronic Data Capture) tool, ${ }^{9,10}$ a secure, web-based software platform designed to support data capture for research studies, providing (1) an intuitive interface for validated data capture; (2) audit trails for tracking data manipulation and export procedures; (3) automated export procedures for seamless data downloads to common statistical packages; and (4) procedures for data integration and interoperability with external sources.

Pilot study patients' pain scores were collected postoperatively through a survey administered at the postoperative visit 7-10 days after surgery that asked patients to recall postoperative day one and week one pain scores. The historical cohort patients' pain scores were collected from a nursing phone call the day after surgery, and this postoperative day one pain score is usually documented in the medical record. Moderate to severe pain was defined as at least 4 on a 10-point scale, and severe pain was defined as at least a 7 on a 10-point scale. Pain scores were further analyzed with respect to lumpectomy type and axillary management. Opioids prescribed in-hospital and at discharge were compared using oral morphine equivalents (OMEs) based on the Centers for Disease Control and Prevention (CDC) mobile application (app). ${ }^{11}$

Statistical analyses were performed using SAS ${ }^{\circledR}$ software, version 9.4 (SAS Institute Inc., Cary, NC, USA) and outsourced to Peter Flom Consulting. Descriptive statistics were compared and postoperative pain scores were analyzed using the Kruskal-Wallis test. Proportions with 95\% exact confidence interval were estimated and a $p$ value $<$ 0.05 was considered statistically significant.
FIG. 1. Preoperative, intraoperative, and postoperative elements of the opioid-sparing multimodal analgesia protocol for patients undergoing lumpectomy. $A P A P$ acetaminophen, GABA gabapentin, $I V$ intravenous
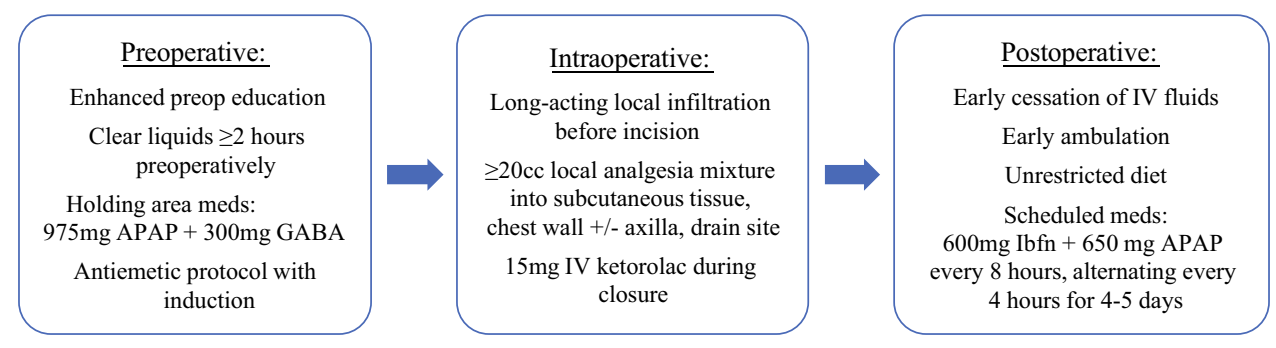
FIG. 2. Study cohort diagram

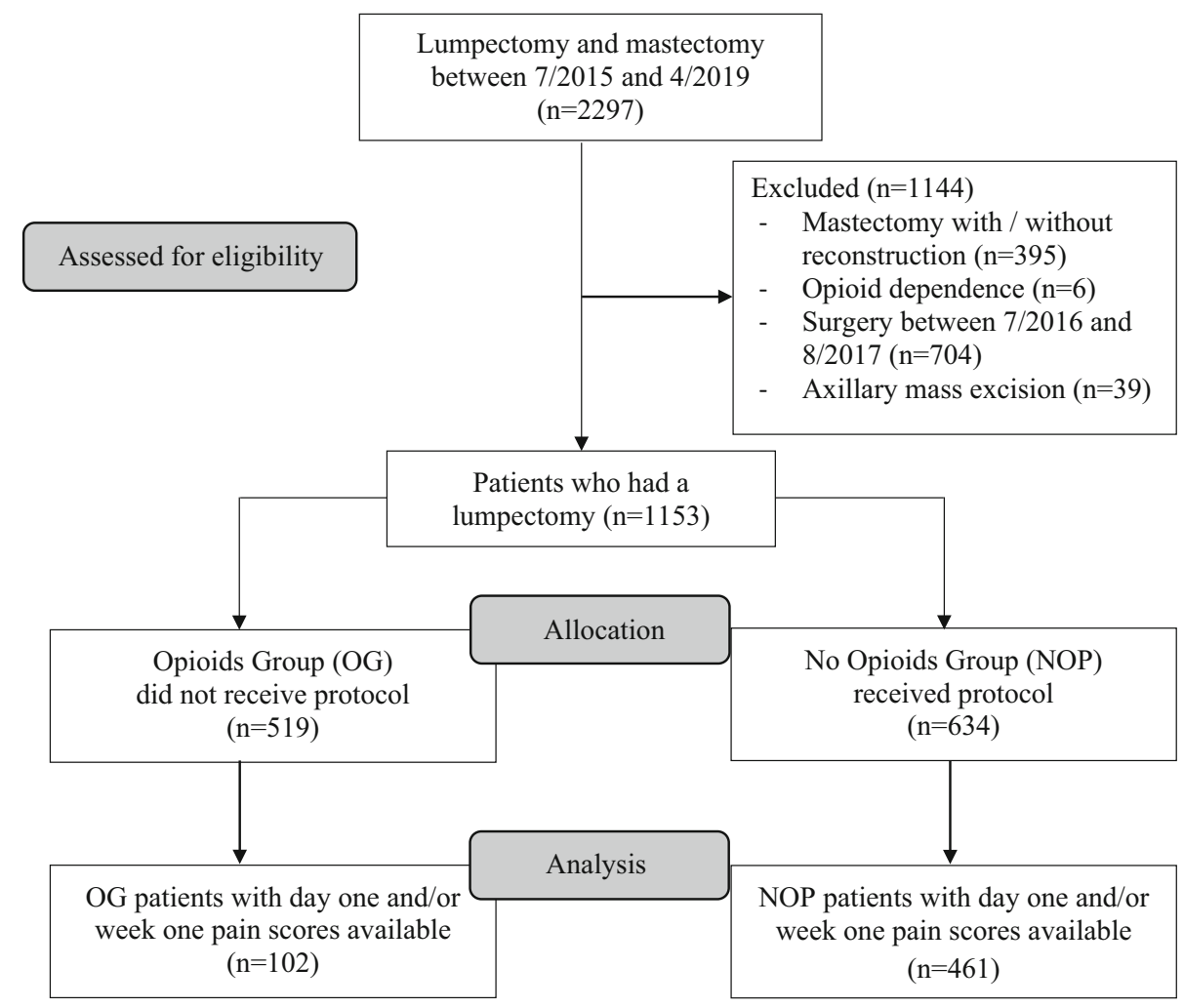

\section{RESULTS}

A combined contemporary and historical cohort included 1153 patients who underwent lumpectomy. Overall, 634 patients received the protocol (NOP) and 519 patients did not (OG); 563 patients had pain scores available for analysis (Fig. 2).

The groups were similar with regard to comorbidities, tobacco use, and history of prior breast cancer (Table 1), although NOP patients were slightly older and more likely to be obese. A significantly greater number of patients in the NOP cohort underwent wireless lumpectomies and significantly fewer patients in the OG cohort underwent axillary surgery.

The majority of the NOP group received all elements of the protocol, including preoperative oral medication, intraoperative ketorolac, and intraoperative liposomal bupivacaine. Most lumpectomy patients did not require postoperative opioids prior to discharge, regardless of protocol receipt. OG patients were discharged with a median of 90 OMEs (range 0-360), while the NOP patients were discharged with no OMEs $(p<0.001)$ (Table 2).

Postoperative pain scores were consistently lower in patients who received the opioid- sparing (NOP) protocol. When analyzed as a whole, NOP patients reported significantly lower postoperative day one and week one pain scores. With respect to lumpectomy type, this significance persisted for both the wire-localized and wireless localization lumpectomy groups. With regard to axillary management, patients who received sentinel node biopsy or no axillary surgery in the NOP group reported significantly lower pain scores than those in the OG cohort, but a comparison with those undergoing axillary dissections was limited by smaller numbers (Table 3 ).

While median pain scores were low regardless of protocol, large reductions in risk of clinically significant pain were seen in patients who received the opioid-sparing (NOP) protocol. On postoperative day one, NOP patients were significantly less likely to report moderate to severe (at least 4/10) and severe pain (at least 7/10). While this risk reduction persisted at postoperative week one, it was not statistically significant (Table 4).

Notably, the risk of moderate to severe pain on postoperative day one was lower in patients undergoing wirelocalized lumpectomies who received the opioid-sparing multimodal analgesia protocol, but this was not appreciated in those undergoing lumpectomy who did not have wire localization (horizontal $p$-values in Table 5). In fact, lumpectomies without wire localization did not have a significantly lower risk of persistent postoperative pain at day one and week one when compared with wire-localized lumpectomies (vertical $p$ values in Table 5). Not surprisingly, even with the NOP protocol, those who had axillary surgery were more likely to report moderate to severe pain at both timepoints after surgery (vertical $p$ values in Table 5). 
TABLE 1. Demographics and clinical characteristics

\begin{tabular}{|c|c|c|c|}
\hline & $\begin{array}{l}\text { Opioids group } \\
{[n=519]}\end{array}$ & $\begin{array}{l}\text { No opioids group } \\
{[n=634]}\end{array}$ & $p$ value \\
\hline Female & $513(98.8)^{\mathrm{a}}$ & $630(99.4)$ & 0.339 \\
\hline Median age, years (range) & $54(12-92)$ & $58(14-104)$ & 0.008 \\
\hline Median BMI (range) & $27.9(15-53)$ & $28.7(14-58)$ & 0.068 \\
\hline Obese patients $(\mathrm{BMI} \geq 30)$ & $189(36.4)$ & $273(43.1)$ & 0.022 \\
\hline \multicolumn{4}{|l|}{ Comorbidities } \\
\hline Any comorbidity & $270(52.0)$ & $348(54.9)$ & 0.332 \\
\hline Cardiovascular disease & $260(50.3)$ & $334(52.8)$ & 0.404 \\
\hline Diabetes & $104(20.1)$ & $109(17.2)$ & 0.209 \\
\hline Tobacco use & & & 0.602 \\
\hline No/never & 404 (77.9) & $483(76.2)$ & \\
\hline Current/past & $110(21.2)$ & $147(23.2)$ & \\
\hline Previous breast cancer & $34(6.6)$ & $52(8.2)$ & 0.302 \\
\hline \multicolumn{4}{|l|}{ Lumpectomy type } \\
\hline Wire localization & $351(67.6)$ & $245(38.6)$ & $<\mathbf{0 . 0 0 1}$ \\
\hline Wireless localization & $22(4.2)$ & $227(35.8)$ & $<\mathbf{0 . 0 0 1}$ \\
\hline Excisional biopsy & $111(21.4)$ & $99(15.6)$ & 0.012 \\
\hline Re-excision & $35(6.7)$ & $63(9.9)$ & 0.053 \\
\hline Proportion with malignancy & $254(48.9)$ & $378(59.6)$ & $<\mathbf{0 . 0 0 1}$ \\
\hline Bilateral surgery & $22(4.2)$ & $40(3.5)$ & 0.121 \\
\hline \multicolumn{4}{|l|}{ Axillary management } \\
\hline None & $363(69.9)$ & $391(61.7)$ & 0.003 \\
\hline Sentinel node biopsy & $136(26.2)$ & $210(33.1)$ & 0.011 \\
\hline Axillary dissection & $20(3.9)$ & $33(5.2)$ & 0.276 \\
\hline
\end{tabular}

Significant $p$ values are given in bold

Data are expressed as $n(\%)$ unless otherwise specified

$B M I$ body mass index
TABLE 2. Protocol adherence and prescribed opioids

\begin{tabular}{llll}
\hline & Opioids group $[n=519]$ & No opioids group $[n=634]$ & $p$ value \\
\hline Perioperative management & & & \\
Preoperative acetaminophen & $12(2.2)^{\mathrm{a}}$ & $532(97.8)$ & $<\mathbf{0 . 0 0 1}$ \\
Preoperative gabapentin & $14(2.6)$ & $525(97.4)$ & $<\mathbf{0 . 0 0 1}$ \\
Intraoperative ketorolac & $27(5.7)$ & $450(94.3)$ & $<\mathbf{0 . 0 0 1}$ \\
Intraoperative liposomal bupivacaine & $9(1.5)$ & $574(98.5)$ & $<\mathbf{0 . 0 0 1}$ \\
Median inpatient OMEs (range) & $0(0-97.5)$ & $0(0-27.5)$ & $<\mathbf{0 . 0 0 1}$ \\
Mean inpatient OMEs & 1.5 & 0.5 & $<\mathbf{0 . 0 0 1}$ \\
Median discharge OMEs (range) & $90(0-360)$ & $0(0-150)$ & $<\mathbf{0 . 0 0 1}$ \\
Mean discharge OMEs [SD] & $112.5[74.6]$ & $5.1[20.0]$ & $<\mathbf{0 . 0 0 1}$ \\
\hline
\end{tabular}

Significant $p$ values are given in bold

Data are expressed as $n(\%)$ unless otherwise specified

$O M E s$ oral morphine equivalents, $S D$ standard deviation

\section{DISCUSSION}

Patients undergoing localized lumpectomy who received the multimodal opioid-sparing protocol were found to have superior postoperative pain control when compared with patients who received traditional care and a routine opioid prescription at discharge. The opioid-sparing protocol provided superior pain control for patients undergoing both wireless and wire-localized lumpectomies, and for those who received no axillary surgery or sentinel node biopsy. 
TABLE 3. Postoperative pain scores by surgery type and axillary management

\begin{tabular}{|c|c|c|c|}
\hline & Opioids group $[n=102]$ & No opioids group [ $n=461]$ & $p$ value \\
\hline \multicolumn{4}{|l|}{ Day one pain scores } \\
\hline Lumpectomy (all) & $2(0-10), n=102^{\mathrm{a}}$ & $0(0-10), n=461$ & $<\mathbf{0 . 0 0 1}$ \\
\hline Wire localization & $2(0-10), n=61$ & $0(0-9), n=181$ & 0.002 \\
\hline Wireless localization & $2(0-10), n=18$ & $0(0-10), n=157$ & 0.002 \\
\hline Excisional biopsy & $0(0-9), n=15$ & $0(0-10), n=75$ & 0.777 \\
\hline Re-excision & $0(0-3), n=8$ & $0(0-10), n=48$ & 0.811 \\
\hline \multicolumn{4}{|l|}{ Axillary management } \\
\hline Sentinel node biopsy & $2(0-8), n=21$ & $0(0-10), n=147$ & $\mathbf{0 . 0 2 0}$ \\
\hline Axillary dissection & $0(0-9), n=5$ & $2(0-10), n=23$ & 0.611 \\
\hline Any axillary surgery & $2(0-9), n=26$ & $0(0-10), n=170$ & 0.073 \\
\hline No axillary surgery & $2(0-10), n=76$ & $0(0-10), n=291$ & $<\mathbf{0 . 0 0 1}$ \\
\hline \multicolumn{4}{|l|}{ Week one pain scores } \\
\hline Lumpectomy (all) & $1(0-8), n=95$ & $0(0-10), n=398$ & $<0.001$ \\
\hline Wire localization & $0(0-8), n=57$ & $0(0-9), n=159$ & 0.006 \\
\hline Wireless localization & $2(0-8), n=17$ & $0(0-10), n=131$ & $<0.001$ \\
\hline Excisional biopsy & $0(0-7), n=15$ & $0(0-8), n=66$ & 0.334 \\
\hline Re-excision & $0(0-2), n=6$ & $0(0-8), n=42$ & 0.544 \\
\hline \multicolumn{4}{|l|}{ Axillary management } \\
\hline Sentinel node biopsy & $1(0-8), n=19$ & $0(0-10), n=124$ & $\mathbf{0 . 0 3 7}$ \\
\hline Axillary dissection & $0(0-8), n=5$ & $0(0-7), n=17$ & 0.963 \\
\hline Any axillary surgery & $1(0-8), n=24$ & $0(0-10), n=141$ & 0.060 \\
\hline No axillary surgery & $0(0-8), n=71$ & $0(0-10), n=257$ & $<0.001$ \\
\hline
\end{tabular}

Significant $p$ values are given in bold

Data are expressed as median (range), $n$

TABLE 4. Rates of clinically significant pain among study groups

\begin{tabular}{|c|c|c|c|}
\hline & $\begin{array}{l}\text { Opioids group } \\
{[n=102]}\end{array}$ & $\begin{array}{l}\text { No opioids group } \\
{[n=461]}\end{array}$ & $p$ value \\
\hline \multicolumn{4}{|l|}{ Postoperative day one } \\
\hline Moderate to severe pain ${ }^{a}$ & $35(34.3)$ & $88(19.1)$ & $<0.001$ \\
\hline \multirow[t]{2}{*}{ Severe pain ${ }^{\mathrm{b}}$} & $16(15.7)$ & $32(6.9)$ & 0.004 \\
\hline & Opioids group $[n=95]$ & $\begin{array}{l}\text { No opioids group } \\
(n=398)\end{array}$ & $p$ value \\
\hline \multicolumn{4}{|l|}{ Postoperative week one } \\
\hline Moderate to severe pain & $20(21.1)$ & $57(14.3)$ & 0.104 \\
\hline Severe pain & $5(5.3)$ & $17(4.3)$ & 0.674 \\
\hline
\end{tabular}

Significant $p$ values are given in bold

Data are expressed as $n(\%)$

${ }^{a}$ Moderate to severe pain: $4-10$ on a 10 -point scale

${ }^{\mathrm{b}}$ Severe pain: $7-10$ on a 10 -point scale

The subset analysis revealed that the protocol resulted in better pain control in patients undergoing sentinel node biopsy. Patients undergoing axillary surgery were more likely to report clinically significant pain at postoperative day one and week one when compared with those who did not receive axillary surgery, regardless of protocol. This is in line with prior reports documenting increased pain in patients receiving axillary intervention at the time of lumpectomy. ${ }^{12}$ Nevertheless, the NOP patients discharged with scheduled acetaminophen and ibuprofen reported 
TABLE 5. Proportion of patients with moderate to severe pain ${ }^{a}$, subgroup analysis

\begin{tabular}{|c|c|c|c|}
\hline & Opioids group $[n=102]$ & No opioids group [ $n=461]$ & $p$ value (row) \\
\hline \multicolumn{4}{|l|}{ Postoperative day one } \\
\hline \multicolumn{4}{|l|}{ Lumpectomy type } \\
\hline Wire localized $(n=242)$ & 24/61 (39.3) & 36/181 (19.9) & 0.002 \\
\hline Non-wire localized $^{\mathrm{b}}(n=321)$ & $11 / 41(26.8)$ & $52 / 280(18.6)$ & 0.214 \\
\hline$p$ Value (column) & 0.275 & 0.818 & \\
\hline \multicolumn{4}{|l|}{ Axillary management } \\
\hline Any axillary surgery $(n=196)$ & $8 / 26(30.8)$ & $43 / 170(25.3)$ & 0.553 \\
\hline No axillary surgery $(n=367)$ & $27 / 76(35.5)$ & 45/291 (15.5) & $<0.001$ \\
\hline \multirow[t]{2}{*}{$p$ Value (column) } & 0.840 & 0.014 & \\
\hline & Opioids group $[n=95]$ & No opioids group [ $n=398]$ & $p$ value (row) \\
\hline \multicolumn{4}{|l|}{ Postoperative week one } \\
\hline \multicolumn{4}{|l|}{ Lumpectomy type } \\
\hline Wire-localized $(n=216)$ & $11 / 57(19.3)$ & 24/159 (15.1) & 0.460 \\
\hline Non-wire localized ${ }^{\mathrm{b}}(n=277)$ & 9/38 (23.7) & $33 / 239(13.8)$ & 0.115 \\
\hline$p$ Value (column) & 0.797 & 0.812 & \\
\hline \multicolumn{4}{|l|}{ Axillary management } \\
\hline Any axillary surgery $(n=165)$ & $8 / 24(33.3)$ & $31 / 141(22.0)$ & 0.226 \\
\hline No axillary surgery $(n=328)$ & $12 / 71(16.9)$ & $26 / 257(10.1)$ & 0.114 \\
\hline$p$ Value (column) & 0.156 & 0.002 & \\
\hline
\end{tabular}

Significant $p$ values are given in bold

Data are expressed as $n / n(\%)$

${ }^{a}$ Moderate to severe pain: score $4-10$ on a 10 -point scale

${ }^{\mathrm{b}}$ Non-wire localized lumpectomy includes excisional biopsy, re-excision, wireless localization

superior postoperative pain control, despite having a greater proportion of patients who received axillary surgery. This suggests that the opioid-sparing protocol may be more effective than measured in this analysis.

With regard to wire- versus wireless localized lumpectomy, there was not a significant difference in postoperative day one pain scores (Fig. 3). While the present study was not specifically designed to compare pain outcomes in these subgroups, patients undergoing wire localization receive an additional procedure on the day of surgery. This finding is in contrast to a previous randomized clinical trial that found that patients who underwent wire localization reported higher postoperative day one pain scores when compared with those whose procedure included wireless localization techniques. ${ }^{13}$

The present work builds upon prior studies showing that opioid minimization after excisional biopsy and lumpectomy is achievable. Moo et al. recently demonstrated that replacement of the reflex discharge opioid with an NSAID (diclofenac) did not compromise postoperative pain control, and few patients $(1.3 \%)$ who were discharged without opioids subsequently requested a prescription. ${ }^{14}$ In contrast to our results, their study did not find superior postoperative pain control with non-opioid adjuncts. However, they did find low postoperative pain scores without the use of liposomal bupivacaine, which highlights the generalizability of their results since use of this local anesthetic is cost prohibitive to many.

Successfully addressing acute postoperative pain leads to a decreased risk of persistent pain, and patients undergoing curative-intent cancer surgery are particularly vulnerable to chronic pain. ${ }^{15-18}$ Chronic pain, defined as extending beyond the 3-month time point from the intervention, disrupts sleep patterns and daily activities, lowers quality of life, and decreases the likelihood of maintaining or returning to employment. ${ }^{19-21}$ While most patients who experience persistent postoperative pain will improve over a 3-year period, 1 in 10 will develop new chronic opioid use after receiving a physician prescription. ${ }^{3,22,23}$

Opioids have traditionally been recognized as an integral part of cancer pain management. ${ }^{24,25}$ While they are known to cause constipation, central nervous system disturbances, and disruption in endogenous opioid pathways, they may also negatively impact oncologic outcome. ${ }^{26,27}$ Preclinical studies have shown that opioids administered at the time of cancer surgery can be deleterious to the normal 
FIG. 3. Subgroup analysis according to localization technique and axillary management. $N S$ non-significant
Moderate to Severe Pain: Postoperative Day One

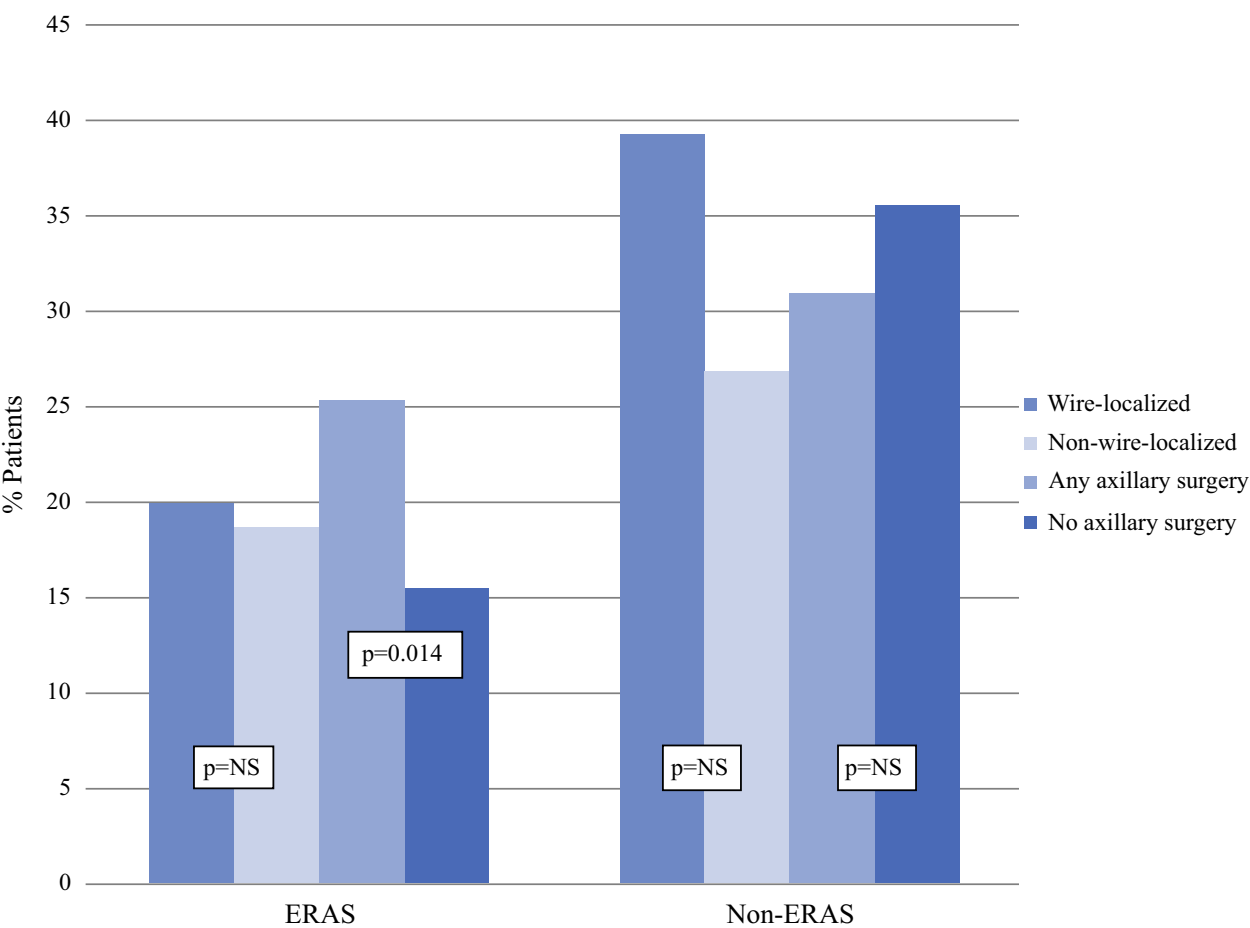

anti-tumor immune response and give rise to worse outcomes through immune system disruption, decreasing the activity of natural killer (NK) cells and dysregulating cellular apoptosis. ${ }^{28-30}$ In addition, studies utilizing murine models have demonstrated morphine-induced tumor progression in breast cancer cell lines treated with opioids. ${ }^{31}$ This oncogenic effect due to vascular neogenesis was not seen in the cells treated with naloxone, a $m u$ opioid nonselective receptor antagonist. ${ }^{32}$ Morphine has also been linked to an increase in breast cancer lung metastasis in similar models. ${ }^{33}$

The relationship between opioids and oncologic outcomes was also investigated in human studies. A Surveillance, Epidemiology, and End Results (SEER) database study including 42,151 patients who underwent colon cancer surgery showed that patients who received non-opioid epidural anesthesia had a significantly longer overall survival compared with patients who received systemic opioid analgesia. ${ }^{34}$ Two retrospective studies including patients with non-small cell lung cancer undergoing curative-intent surgery found an association between perioperative opioid use, increased recurrence rates, and decreased overall survival. ${ }^{35,36}$ Pooled data from two randomized clinical trials were reported by Janku et al. and revealed that treatment with a peripherally active $т и$ opioid receptor antagonist improved overall survival in end-stage cancer patients. ${ }^{37}$ Combined with the preclinical data, these studies provide a large body of evidence that opioids have the potential to worsen oncologic outcomes.
In contrast, NSAIDs are effective non-opioid adjuncts that reduce postoperative pain after surgery and may also have antineoplastic effects. ${ }^{38-42} \mathrm{~A}$ randomized clinical trial comparing diclofenac with placebo after mastectomy with reconstruction reported that patients experienced significantly less pain on the day after surgery, which translated into lower postoperative opioid consumption. ${ }^{43}$ Preclinical studies have suggested that NSAIDs may improve oncologic outcomes. Surgery itself induces an inflammatory state that may lead to dormancy escape of micrometastases, but preoperative ketorolac has been shown to mitigate these effects through cyclooxygenase (COX)- 1 and COX-2 inhibition. ${ }^{44-46}$ Furthermore, two retrospective studies of women undergoing breast cancer surgery found that those who received intraoperative parenteral ketorolac had a significantly lower rate of cancer recurrence. Notably, this benefit was even greater in obese patients. ${ }^{47,48}$

The present study constitutes a comprehensive comparison of patient-reported pain outcomes after lumpectomy. Incorporation of a large historical multi-surgeon cohort from a culturally diverse area lends to the generalizability of results. The analysis is timely in that the ongoing opioid crisis continues to evolve. The CDC released a preliminary report revealing a $5 \%$ increase in the rate of opioid overdose deaths in 2019, and a more recent report by the American Medical Association (AMA) describes an increase in opioid-related mortality in more than 40 states since the onset of the coronavirus disease 2019 (COVID-19) pandemic. $^{49,50}$ 
While the study groups were demographically similar, more patients in the OG cohort underwent wire-localized lumpectomy compared with wireless localizations, but this is likely a function of timing since a large proportion of OG patients from the historical cohort predated the widespread use of wireless technologies. Patients in the NOP group were surveyed 7-10 days after their surgery, and therefore their report of previous pain on postoperative day one may be subject to recall bias. Non-randomization also makes it difficult to discern whether a particular element or a combination of the protocol elements led to the low pain scores reported in the NOP patients. Similarly, NOP patients who received the long-acting local analgesia may have had similar results without the preoperative medications, intraoperative NSAID, and postoperative non-opioid adjuncts. Subsequent prospective work should be designed to answer this question since the inclusion of this particular long-acting local anesthetic may be cost prohibitive for some.

Several studies have found that prescriptions are the main source of diverted opioids leading to overdose. ${ }^{3}$ Approximately $80 \%$ of patients with opioid use disorder had a personal opioid prescription before their abuse diagnosis and $10 \%$ report accessing opioids through a family member's prescription. ${ }^{51,52}$ Widespread implementation of opioid-sparing multimodal analgesia protocols eliminating the routine opioid prescription at discharge will undoubtedly lead to an unprecedented reduction in the opioids available for diversion.

Future investigations should focus on addressing patients at increased risk for chronic pain and therefore opioid dependence. Younger age, pre-existing pain syndromes, anxiety, and high body mass index have all been associated with increased postoperative pain. ${ }^{53-57}$ Incorporating screening measures to identify these patients preoperatively would allow surgeons to refer patients to the appropriate subspecialists and may highlight necessary adjustments in the multimodal protocol.

\section{CONCLUSION}

Implementation of an opioid-sparing multimodal analgesia protocol for patients undergoing lumpectomy is feasible and results in the elimination of routine postoperative opioid prescribing. Furthermore, incorporation of the protocol led to superior postoperative pain control when compared with similar patients who were routinely discharged with opioids, demonstrating that opioid administration is not a requisite for excellent pain control after lumpectomy. Looking to the future, opioid minimization may prove further beneficial given that perioperative opioids may worsen cancer outcomes.
ACKNOWLEDGMENT The authors would like to acknowledge the Maimonides Medical Center anesthesiologists, perioperative staff, general surgery residents, anesthesia residents, pharmacists, and physician assistants as well as the administrative staff of the Maimonides Breast Center who facilitated the introduction of the opioidsparing protocol and were integral to the success of the program.

FUNDING This research did not receive any funding from agencies in the public, commercial, or not-for-profit sectors.

DISCLOSURE Patrick Borgen, Kristin E. Rojas and Donna-Marie Manasseh have received speaker's honoraria from Pacira Pharmaceuticals, Inc. Claudya Morin, Yamini Patel, Munazza Javid, Sarah E. Tevis, Thais Fortes, Peter Flom, and Charusheela Andaz report no relevant commercial, financial, consultant, or institutional conflicts of interest.

\section{REFERENCES}

1. American Cancer Society. Breast cancer facts and figures 2019-2020. Atlanta: American Cancer Society, Inc.; 2019.

2. Hill MV, McMahon ML, Stucke RS, Barth RJ Jr. Wide variation and excessive dosage of opioid prescriptions for common general surgical procedures. Ann Surg. 2017;265(4):709-14.

3. Compton WM, Boyle M, Wargo E. Prescription opioid abuse: problems and responses. Prev Med. 2015;80:5-9.

4. Caraceni A, Hanks G, Kaasa S, et al. Use of opioid analgesics in the treatment of cancer pain: evidence-based recommendations from the EAPC. Lancet Oncol. 2012;13:e58-68.

5. Vella-Brincat J, Macleod AD. Adverse effects of opioids on the central nervous systems of palliative care patients. J Pain Palliat Care Pharmacother. 2007;21(1):15-25.

6. Rojas K, Fortes T, Flom P, et al. Intraoperative ketorolac use does not increase the risk of bleeding in breast surgery. Ann Surg Oncol. 2019;26(10):3368-73.

7. Rojas K, Manasseh DM, Flom PL, et al. A pilot study of a breast surgery enhanced recovery after surgery (ERAS) protocol to eliminate narcotic prescription at discharge. Breast Cancer Res Treat. 2018;171(3):621-6.

8. Rojas K, Fortes TA, Flom PL, et al. Mastectomy is no longer an indication for postoperative opioid prescription at discharge. Am J Surg. 2019;218(4):700-5.

9. Harris PA, Taylor R, Thielke R, et al. Research electronic data capture (REDCap) - a metadata-driven methodology and workflow process for providing translational research informatics support. J Biomed Inform. 2009;42(2):377-81.

10. Harris PA, Taylor R, Minor BL, et al. The REDCap consortium: building an international community of software partners. $J$ Biomed Inform. 2019;95:103208.

11. Guideline Resources: CDC Opioid Guideline Mobile App. http:// www.CDC.gov. 29 July 2019.

12. Peuckmann V, Ekholm O, Rasmussen NK, et al. Chronic pain and other sequelae in long- term breast cancer survivors: nationwide survey in Denmark. Eur J Pain. 2009;13(5):478-85.

13. Moreno M, Wiltgen JE, Bodanese B, et al. Radioguided breast surgery for occult lesion localization-correlation between two methods. J Exp Clin Cancer Res. 2008;27:29.

14. Moo TA, Assel M, Yeahia R, Nierstedt R, et al. Routine opioid prescriptions are not necessary after breast excisional biopsy or lumpectomy procedures. Ann Surg Oncol. 2021;28(1):303-9. 
15. Tasmuth T, Kataja M, Blomqvist C, et al. Treatment-related factors predisposing to chronic pain in patients with breast cancer: a multivariate approach. Acta Oncol. 1997;36:625-30.

16. Macrae WA. Chronic pain after surgery. $\mathrm{Br} J$ Anaesth. 2001;87:88-98.

17. Perkins FM, Kehlet H. Chronic pain as an outcome of surgery: a review of predictive factors. Anesthesiology. 2000;93:1123-33.

18. Kehlet H, Dahl JB. Anaesthesia, surgery, and challenges in postoperative recovery. Lancet. 2003;362:1921-8.

19. Hamood R, Hamood H, Merhasin I, Keinan-Boker L. Chronic pain and other symptoms among breast cancer survivors: prevalence, predictors, and effects on quality of life. Breast Cancer Res Treat. 2018;167(1):157-69.

20. Gahm J, Wickman M, Brandberg Y. Bilateral prophylactic mastectomy in women with inherited risk of breast cancerprevalence of pain and discomfort, impact on sexuality, quality of life and feelings of regret two years after surgery. Breast. 2010;19(6):462-9.

21. Verbelen H, Tjalma W, Meirte J, Gebruers N. Long-term morbidity after a negative sentinel node in breast cancer patients. Eur $J$ Cancer Care (Engl). 2019;28(5):e13077.

22. Lee JS, Hu HM, Edelman AL, et al. New persistent opioid use among patients with cancer after curative-intent Surgery. J Clin Oncol. 2017;35:4042-9.

23. Antao L, Shaw L, Ollson K, et al. Chronic pain in episodic illness and its influence on work occupations: a scoping review. Work. 2013;44(1):11-36.

24. Lötsch J, Ultsch A, Kalso E. Data-science-based subgroup analysis of persistent pain during 3 years after breast cancer surgery: a prospective cohort study. Eur J Anaesthesiol. 2020;37(3):235-46.

25. Paice JA. Cancer pain management and the opioid crisis in America: How to preserve hard-earned gains in improving the quality of cancer pain management. Cancer. 2018;124(12):2491-7.

26. Leppert W. The impact of opioid analgesics on the gastrointestinal tract function and the current management possibilities. Contemp Oncol. 2012;16(2):125-31.

27. Taylor B, Corder G. Endogenous analgesia, dependence, and latent pain sensitization. Curr Top Behav Neurosci. 2014;20:283-325.

28. Byrne K, Levins KJ, Buggy DJ. Can anesthetic-analgesic technique during primary cancer surgery affect recurrence or metastasis? Can J Anaesth. 2016;63:184-92.

29. Shavit Y, Ben-Eliyahu S, Zeidel A, Beilin B. Effects of fentanyl on natural killer cell activity and on resistance to tumor metastasis in rats. Dose and timing study. Neuroimmunomodulation. 2004; 11:255-60.

30. Aich A, Gupta P, Gupta K. Could perioperative opioid use increase the risk of cancer progression and metastases? Int Anesthesiol Clin Fall. 2016;54(4):e1-16.

31. Nguyen J, Luk K, Vang D, et al. Morphine stimulates cancer progression and mast cell activation and impairs survival in transgenic mice with breast cancer. Br J Anaesth. 2014;113(Suppl 1):i4-13.

32. Gupta K, Kshirsagar S, Chang L, et al. Morphine stimulates angiogenesis by activating proangiogenic and survival-promoting signaling and promotes breast tumor growth. Cancer Res. 2002;62:4491-8.

33. Farooqui M, Li Y, Rogers T, et al. COX-2 inhibitor celecoxib prevents chronic morphine- induced promotion of angiogenesis, tumour growth, metastasis and mortality, without compromising analgesia. Br J Cancer. 2007;97:1523-31

34. Cummings K, Xu F, Cummings L, Cooper G. A comparison of epidural analgesia and traditional pain management effects on survival and cancer recurrence after colectomy: a populationbased study. Anesthesiology. 2012;116(4):797-806.
35. Maher D, Wong W, White P, et al. Association of increased postoperative opioid administration with non-small-cell lung cancer recurrence: a retrospective analysis. $\mathrm{Br} J$ Anaesthesia. 2014;113(1):i88-94.

36. Cata J, Keerty V, Keerty D, et al. A retrospective analysis of the effect of intraoperative opioid dose on cancer recurrence after non-small cell lung cancer resection. Cancer Med. 2014;3(4):900-8.

37. Janku F, Johnson L, Karp D, et al. Treatment with methylnaltrexone is associated with increased survival in patients with advanced cancer. Ann Oncol. 2016;27(11):2032-8.

38. Węgorowski P, Stanisławek A, Domżał-Drzewicka R, et al. The effect of pre-emptive analgesia on the level of postoperative pain in women undergoing surgery for breast neoplasm. Contemp Oncol (Pozn). 2016;20(2):158-64.

39. Przesmycki K, Wiater-Kozioł E, Kotarski J, et al. Effect of preemptive pregabalin on pain intensity and morphine requirement after hysterectomy. Anestezjol Intens Ter . 2011;43(1):14-7.

40. Priya V, Divatia JV, Sareen R, Upadhye S. Efficacy of intravenous ketoprofen for pre- emptive analgesia. J Postgrad Med . 2002;48(2):109-12.

41. Raja DC, Shetty AP, Subramanian B, et al. A prospective randomized study to analyze the efficacy of balanced pre-emptive analgesia in spine surgery. Spine J. 2019;19(4):569-77.

42. Sarakatsianou C, Theodorou E, Georgopoulou S, et al. Effect of pre-emptive pregabalin on pain intensity and postoperative morphine consumption after laparoscopic cholecystectomy. Surg Endosc. 2013;27(7):2504-11.

43. Legeby M, Sandelin K, Wickman M, Olofsson C. Analgesic efficacy of diclofenac in combination with morphine and paracetamol after mastectomy and immediate breast reconstruction. Acta Anaesthesiol Scand. 2005;49(9):1360-6.

44. Güç E, Pollard J. Dampening the fire to prevent surgery- and chemotherapy-induced metastasis. $J$ Clin Invest. 2019;129(7):2663-5. https://doi.org/10.1172/JCI129705.

45. Tsujii M, Kawano S, Tsuji S, Sawaoka H, Hori M, DuBois RN. Cyclooxygenase regulates angiogenesis induced by colon cancer cells. Cell. 1998;93(5):705-16.

46. Panigrahy D, Gartung A, Yang J, et al. Preoperative Stimulation of resolution and inflammation blockade eradicates micrometastases. J Clin Invest. 2019;129(7):2964-79.

47. Forget P, Vandenhende J, Berliere M, et al. Do intraoperative analgesics influence breast cancer recurrence after mastectomy? A retrospective analysis. . Anesth Analg. 2010;110(6):1630-5.

48. Desmedt C, Demicheli R, Fornili M, et al. Potential benefit of intra-operative administration of ketorolac on breast cancer recurrence according to the patient's body mass index. $J$ Natl Cancer Inst. 2018;110(10):1115-22.

49. Ahmad FB, Rossen LM, Sutton P. Provisional drug overdose death counts. National Center for Health Statistics; 2020.

50. Issue brief: Reports of increases in opioid-related overdose and other concerns during the COVID pandemic. American Medical Association Advocacy Resource Center. https://www.ama-assn. org/system/files/2020-09/issue-brief-increases-in-opioid-relatedoverdose.pdf. Accessed 2 Oct 2020.

51. Volkow N, McLellan T. Opioid abuse in chronic pain- misconceptions and mitigation strategies. New Engl J Med. 2016;374:1253-63.

52. Shei A, Rice JB, Kirson NY, et al. Sources of prescription opioids among diagnosed opioid abusers. Curr Med Res Opin. 2015;31:779-84.

53. Gartner R, Jensen MB, Nielsen J, et al. Prevalence of and factors associated with persistent pain following breast cancer surgery. JAMA. 2009;302(18):1985-92. 
54. Katz J, Poleshuck EL, Andrus CH, et al. Risk factors for acute pain and its persistence following breast cancer surgery. Pain. 2005;119(1-3):16-25.

55. Wang L, Guyatt GH, Kennedy SA, et al. Predictors of persistent pain after breast cancer surgery: a systematic review and metaanalysis of observational studies. CMAJ. 2016;188(14):E352-61.

56. Morin C, Javid M, Patel Y, et al. Obese patients who receive an opioid-sparing enhanced recovery after surgery (ERAS) protocol are at increased risk of persistent pain after breast surgery. Ann Surg Oncol. 2020;27:4802-9.
57. Divella M, Vetrugno L, Bertozzi S, Seriau L, Carla C, Bove T. Patient-reported pain and other symptoms among breast cancer survivors: prevalence and risk factors. Tumori. 2020;106(6):480-90.

Publisher's Note Springer Nature remains neutral with regard to jurisdictional claims in published maps and institutional affiliations. 